\title{
HEAVY ION BEAM PROBE COORDINATE MAPPING AND CALIBRATION AT WEGA STELLARATOR
}

\author{
Y. Podoba, M. Otte, F. Wagner. \\ Max-Planck-Institut für Plasmaphysik, EURATOM Ass., D-17491 Greifswald, Germany
}

L. Krupnik, A. Zhezhera

Kharkov Institute of Plasma Physics, Kharkov, Ukraine

10.07.2009

\begin{abstract}
The heavy ion beam probe (HIBP) is an established non-perturbing diagnostic for high spatially and temporary resolved measurements of magnetically confined plasma parameters such as potential, density and temperature. These quantities can be determined from the change in the ion beam parameters (charge, intensity and trajectory) passing through a plasma volume due to collisions with electrons and interaction with the confining magnetic field. One of the problems that should be solved during HIBP installation and tuning is the coordinate matching. Conventionally the coordinate mapping of the HIBP measurement point is provided by ray tracing calculations of the ion beam in the magnetic field. However, it is very difficult to include all physical effects
\end{abstract}


and uncertainties in the model. Thus, the result of the calculations may differ from the real probing position. In order to improve the mapping precision of the HIBP installed at the WEGA stellarator an additional measurement of the beam position is provided using a primary beam detector array inside the vacuum vessel. This allows comparing the measured and calculated ion beam positions in order to prove the calculated coordinate precision and include adjustments in the calculation code if necessary. The principle and the results of this calibration, which is not specific to WEGA but could be adapted to other experiments as well, are presented in this work.

Keywords: HIBP calibration, plasma diagnostics, WEGA stellarator

PACS: 52.25.Xz, 52.70.Nc, 52.55.Hc, 52.65.Cc

\section{Introduction}

A Heavy Ion Beam Probe (HIBP) is a unique diagnostic, which provides direct nonperturbing and localised plasma parameters measurements in toroidal magnetic confinement ${ }^{1,2}$ and $\operatorname{arc}^{3}$ experiments. The diagnostic principle is based on the difference in the Larmor radii of highly energetic heavy ions at different ionization states. A singly charged ion beam (primary beam) is injected into the plasma across the confining magnetic field. Some particles are ionized due to collisions with plasma particles producing a fan of doubly ionized secondary ions (secondary beam). Information on plasma parameters in the ionisation point can be obtained from the characteristics of the secondary ions. The plasma potential $\Phi_{p}$ is deduced from differences between the primary and secondary beam energy. The HIBP can also be used to measure the electron 
density $n_{e}$, the electron temperature $T_{e}$ from the intensity of the secondary beam and the poloidal magnetic field $B_{\theta}$ from the initial beam trajectory deviation. The HIBP on WEGA stellarator was installed in $2006-2007^{4}$. First single point potential measurements were obtained in $2007^{5}$.

One of the main problems that should be solved during HIBP installation and tuning is the coordinate matching. The task is to establish a correspondence between the real geometrical position of the ionisation point and the control parameters of the HIBP diagnostic, such as the energy of the primary beam and the sweeping voltage of the deflecting plates, used to vary the initial primary beam position. Conventionally this is accomplished by using ray tracing calculations of the heavy ions. However, the idealization of the computational model may introduce a mismatch between the calculated and the real position of the measurements. In this article, we present a calibration method, which uses experimental data for the validation of HIBP ray tracing calculations.

\section{HIBP on WEGA}

WEGA is a medium sized classical five period stellarator with $l=2$ poloidal symmetry. It has a major radius of $R=72 \mathrm{~cm}$ and a maximum average plasma radius of $a=11 \mathrm{~cm}^{6}$. In HIBP experiments the plasma is produced and heated by a gyrotron with a power of $P=10 \mathrm{~kW} \mathrm{cw}$ at a frequency of $28 \mathrm{GHz}$. Magnetic field strength is set at $|B|=0.489 \mathrm{~T}$ providing a resonant conditions for X2 mode heating ${ }^{7}$. As working gases $\mathrm{H}_{2}, \mathrm{He}$ and $\mathrm{Ar}$

are used. Plasmas typically have a central density of about $n_{e} \sim 5 \times 10^{18} \mathrm{~m}^{-3}$ and a temperature of about $T_{e}>25 \mathrm{eV}$. However, in the OXB regime ${ }^{8}$ a density of 
$n_{e}>1 \times 10^{19} \mathrm{~m}^{-3}$ could be reached.

For the HIBP singly charged sodium ions $\left(\mathrm{Na}^{+}\right)$are used as a primary beam with a current of $I_{b}=25 \mu A$ and an energy of $E=39.5 \mathrm{keV}$. These parameters are optimal for the nominal toroidal magnetic field value. The beam width of about $\sim 5 \mathrm{~mm}$ in the measurement point along with the geometrical properties of WEGA provides a spatial resolution of plasma parameters measurements from 15 to $7 \mathrm{~mm}$ depending on the position in the plasma. The time resolution of $20 \mu \mathrm{s}$ is limited by the electronics of the data acquisition system. The covered radial range of the measurements is $0.25<r / a<1$, where $r$ is the distance to the plasma centre $(r / a=0)$. The plasma centre itself is not accessible for the HIBP due to geometrical limitations of the installation.

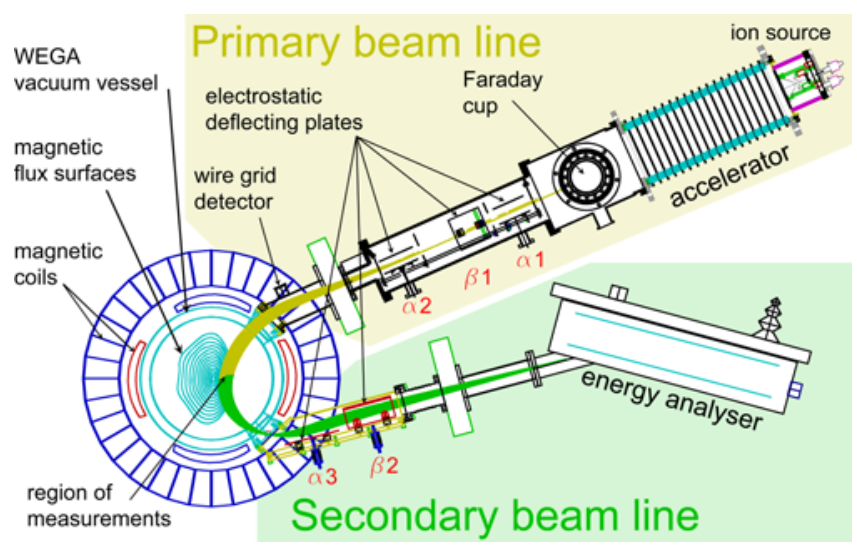

Fig. 1. (colour online) Scheme of the HIBP installed at the WEGA stellarator.

The HIBP system consists of a primary and a secondary beam-line (Fig.1). In the primary 
beam-line, $\mathrm{Na}^{+}$beam is accelerated and focused by an electrostatic field in the accelerator tube. The beam point and angle of incidence in the plasma are controlled by electrostatic deflecting plates $\alpha 1, \alpha 2$ and $\beta 1$ and are monitored by a wire grid detector at the output of the primary beam line. In the plasma $\mathrm{Na}^{+}$ions are deflected by the WEGA confining magnetic field. Due to collisions with plasma ions, electrons, and background neutrals, a part of the primary ions is ionised and turned into double-charged $\mathrm{Na}^{++}$. Secondary ions form a fan which reaches the secondary beam-line entrance (Fig.2). In the secondary beam-line electrostatic plates $\alpha 3$ and $\beta 2$ deflect that part of the secondary fan originating from a specific sample volume to the energy analyser entrance. Voltages on $\alpha 3$ and $\beta 2$ deflecting plates define the position of the sample volume along the primary beam trajectory. In this way, the voltages on all deflecting plates define the position of the sample volume in space.

The secondary beam to be analysed is singled out from the fan of secondary particles by the energy analyser entrance slit. The energy and the total current of the secondary ions originating from the selected sample volume are measured in a Proca-Green design ${ }^{9}$ energy analyser, which is installed at the end of the secondary beam line (Fig.1).

\section{CALIBRATION TECHNIQUE}




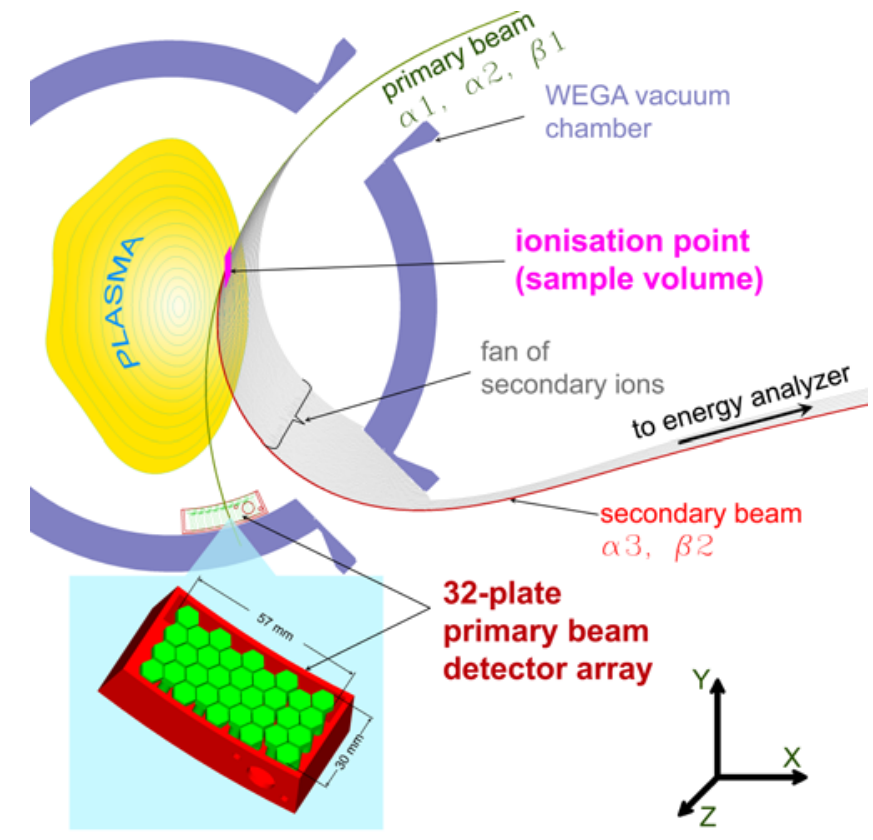

Fig. 2. (colour online) HIBP arrangement with the additional primary beam detector array. The plasma shape is shown for $I_{t o r}=3366 A$ in the toroidal coils and $I_{h e l}=4711 A$ in the helical coils respectively. $\alpha 1, \alpha 2$, and $\beta 1$ plates define the insertion position and the angle of primary beam, $\alpha 3$ and $\beta 2$ plates control the sample volume position along the primary beam.

As mentioned before the coordinate of the ionization point is calculated utilizing a ray tracing code. This code uses the magnetic configuration of WEGA together with the electrostatic fields of the HIBP deflecting system for the calculation of the primary and secondary ion trajectories based on a Runge-Kutta method. However, the code uses the ideal geometry of the installation. In reality, assembly errors result in a mismatch between the calculation results and real ionization point position. 
In order to optimize the calculated coordinates the ion beam position should be measured independently in an appropriate reference frame. One possibility is to use a movable detector and a gas (or target) ionization method ${ }^{10}$ as used at $\mathrm{JFT}^{-2 \mathrm{M}^{11}}$ or CHS ${ }^{12}$. This technique gives very precise information about the measurement point position, also the potential measurements could be verified and energy analyser calibration performed. However, application of this technique at WEGA is impossible due to limited accessibility of the inner space of the vacuum chamber. Alternative primary beam detector (PBD) method for HIBP geometry validation is used on the WEGA. This method allows only spatial alignment of the primary beam. No calibration of the potential measurements or secondary beam trajectory is possible. However, because of the method simplicity the precise primary beam alignment could be obtained with minimum expenses. Such measurements were successfully performed on various magnetically confining devices where HIBP was installed $\left(\mathrm{LHD}^{1}, \mathrm{MST}^{13}, \mathrm{EBT}^{14}, \mathrm{NBT}^{15}\right)$.

Various types of PBD were used at afire mentioned devices. The advantage of the detector used at WEGA is that it could measure not only the coordinate of the primary beam in poloidal direction, but also the toroidal shift of the beam could be defined. This allows justifying the primary beam line position in poloidal and toroidal direction with very high precision.

Generally PBD gives the information about the final coordinate and shape of the primary beam. In a presence of plasma PBD measurements are complicated because of plasma load on the detector plates. In order to detect the beam in this case the modulation techniques could be used and the beam current could be increased to improve signal to noise ratio. In the case when the magnetic field configuration is fully defined by external 
magnetic coils system the calibration of HIBP by PBD measurements could be provided in "plasma free" experiments where no plasma ignition and heating are performed. This is the case of the stellarators where the plasma current is not affecting the magnetic configuration. This feature of stellarator-like devices is used for PBD calibration measurements at WEGA. No measurements in plasma presence were provided in the frame of this work.

As reference points on WEGA the wire grid detector and plate detectors are used to measure the current when the primary ion beam touches its surface. Initially a set of wiredetectors was installed at the output of the primary beam before the ion beam enters the WEGA chamber (Fig.1). These detectors provide information on the beam position at the end of the primary beam-line before entering the magnetic confinement region. In order to obtain full information on the primary beam trajectory it is necessary to measure its position at another point which is far enough from the wire-detector to provide a good base line for the measurements. For this purpose, an array of 32 precisely positioned hexagonal-shape detector plates was installed inside the WEGA chamber (Fig.2). The plates are installed in a stainless steel shielding box to prevent an interaction of the plate array with the edge plasma. The array of the current measuring plates is shielded additionally from heating microwave by a metallic grid. The box is fixed at the bottom of the WEGA vacuum chamber. At this point the primary beam touches the wall during the operation at nominal magnetic field. The range of $57 \mathrm{~mm}$ in radial direction and $30 \mathrm{~mm}$ in toroidal direction is covered with current measuring plates (Fig.2). The shielding box has a bent shape to realize an alignment with respect to the WEGA vacuum chamber wall. These 32 plates are connected to 8 ADC channels through amplifiers with a low pass 
filter as shown in Fig.3. Thus, each channel acquires the sum-current of four spatially separated plates. The typical measured current signals are also shown in Fig.3. One of the eight signals is highlighted (red line). Other signals (grey curves) behave similarly. It is seen that up to four maxima of measured current on one signal could be obtained if the beam touches all four plates connected to the same ADC channel during the scan process. The maxima of the current signal are attributed to the four connected plates via ray tracing calculations based on the experimental conditions.

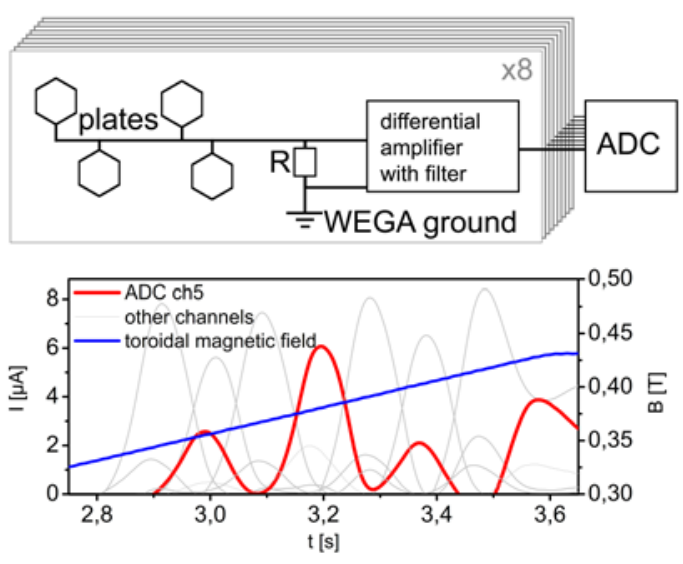

\section{Fig. 3. (colour online) Simplified scheme of the array plates connection and typical signals obtained during a magnetic field scan.}

Thus, the array provides the second reference point for the cross-checking of the calculated positions. The precision of these measurements is given by the beam diameter of $\sim 7 \mathrm{~mm}$ in the region of the array and the plate size of $\sim 6 \mathrm{~mm}$. Thus, assuming a 
Gaussian current distribution in the primary beam the position of the beam centre is defined with a precision of $\sim 7 \mathrm{~mm}$. Practically, real measurement precision is higher because during the scan measurements the primary beam passes the plate and the maximum of the current corresponds to the moment when the beam hits the centre of the plate. Consequently, the position of the beam centre is known with a precision of nearly 1 $m m$, which is defined by the PBD array positioning fidelity. However, even this "worst" precision value of $\sim 7 \mathrm{~mm}$ is already enough for a very precise primary beam-line positioning.

The 32-plate detector array at the end of the primary beam trajectory and the wire detector at the output of the primary beam line measure the position of the primary beam simultaneously.

The behaviour of the beam was investigated in experiments with changing magnetic field (toroidal and helical) and varying deflection voltages in the primary beam line.

\section{COORDINATE MAPPING}

In Fig. 4 the footprints of the primary beam on the detector array are shown for a toroidal magnetic field scan (a) and for a helical magnetic field scan with toroidal magnetic field staying constant at nominal value (b). Here, the deflecting voltage at the $\beta 1$ plate is zero which means no toroidal correction of the primary beam. In this case during the pure toroidal magnetic field scan the beam footprint should always be at $\mathrm{z}=0$ as shown in Fig.4 footprint (a) by the dashed arrow.

The coordinate system, shown in this figure, has its origin in the centre of the poloidal cross section where the HIBP is installed with the $\mathrm{x}$-axis directed along the major radius 
of the torus. The y-axis is perpendicular to the equatorial, and the $\mathrm{z}$-axis perpendicular to the poloidal plane.

Also an $\alpha 2$ plate deflecting voltage scan is shown in Fig. 4 by the footprint (c). Both the helical and toroidal magnetic fields are at nominal values in this measurement. Here, the $\beta 1$ voltage is equal to $U=-130 \mathrm{~V}$ which is the optimal value of toroidal beam correction for which the maximum of the measured secondary current is observed in the energy analyzer during the plasma experiments. The footprints calculated by the ray tracing code are shown by dashed arrows, the measured data are shown by solid arrows. 

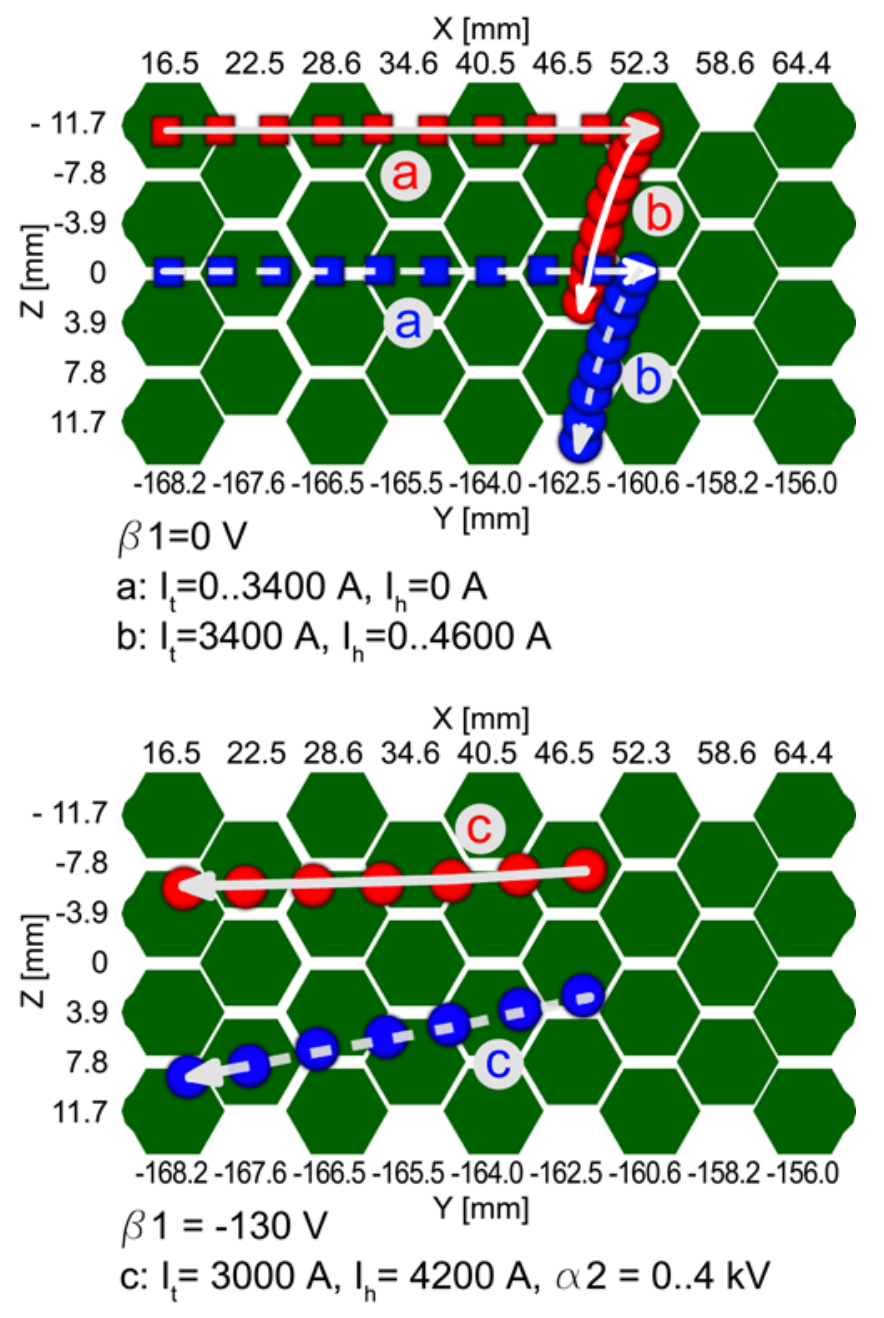

Fig. 4. (colour online) The footprint of the primary beam on the detector array during toroidal magnetic field scan (a) shot \#28585 and helical magnetic field scan (b) shot \#29578. The footprints for the $\alpha 2$ plate voltage scan at nominal magnetic field configuration are shown in (c). Measured positions are red (solid arrows), calculated positions are blue (dashed arrows). Circle and square marks shown on this picture are not representing real primary beam size and shape but purely show the points where the measured coordinates were compared with calculated one. 
The comparison of measured and calculated results exhibits two deviations. One is observed in magnetic field scans as shown in Fig.4 by comparing the footprints (a) and (b). The toroidal position of the primary beam ( $\mathrm{z}$ coordinate) at the array position is shifted by approximately $11 \mathrm{~mm}$. Assumed reason for this is a misalignment of the primary beam incidence angle in comparison to the designed value in z-axis (toroidal) direction (Fig.5a). The difference between the designed and the real value is found to be $\sim 0.4^{\circ}$, which matches the observed deviation of $11 \mathrm{~mm}$ in toroidal direction.

The second deviation has been detected during the $\alpha 2$ voltage scan. Calculations show beam behaviour as depicted in the footprint (c) of Fig.4 (dashed line). The footprint in this case has a certain angle to the $\mathrm{x}$-axis due to the influence of the poloidal magnetic field component. However, the measured beam trace follows almost parallel the radial direction (solid line). Such an inconsistency of measured and calculated results can not be explained by only a toroidal angle mismatch. This deviation points to an inaccuracy in the adjustment of the primary deflecting $\alpha 2$ plates in horizontal direction (Fig.5b). If the $\alpha 2$ deflecting plates are not ideally aligned in the horizontal plane the ion beam will be deflected not only in the $x-y-p l a n e$, but also in $\mathrm{z}$ (toroidal) direction. In the WEGA case this parasitic toroidal shift just compensates the influence of the poloidal magnetic field component on the primary beam trajectory. As a result, the measured primary beam footprint during the $\alpha 2$ scan is nearly parallel to the radial direction, which, in turn, implies that the HIBP measurement points deviate less from the poloidal cross section. The angle between the deflecting plate surface and the horizontal plane in WEGA is found to be nearly $2.3^{\circ}$. Both deviations lead to the toroidal beam position mismatch. 
However, the radial position of the primary beam (x coordinate of the beam) is found to be consistent with the calculated values in the range of the measurement's precision. Therefore, no additional correction of the calculations is necessary for the radial coordinate of the ionization point.

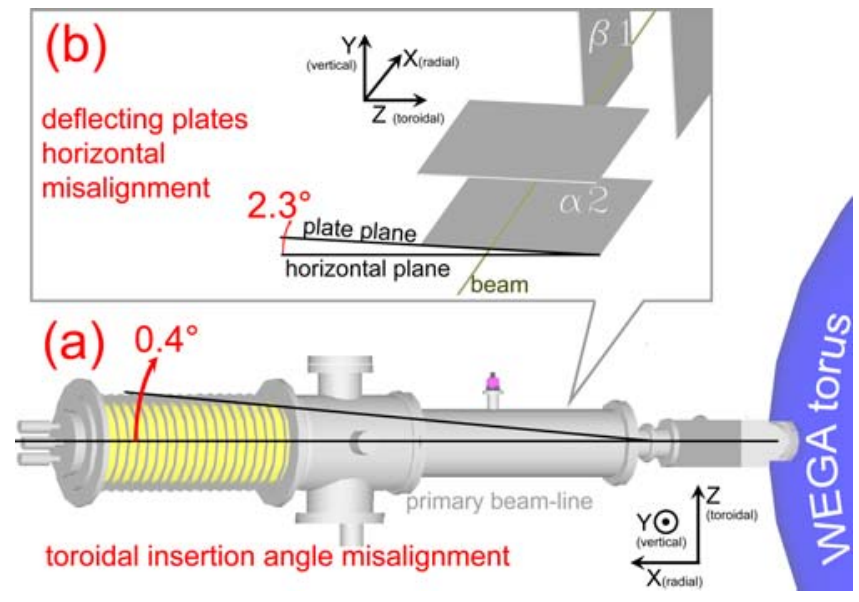

Fig. 5. (colour online) Geometrical misalignment of the primary beam line toroidal angle (a) and deflecting plates horizontal position (b)

\section{CONCLUSIONS}

The primary beam detection technique of HIBP coordinate mapping validation was developed and applied on the WEGA stellarator. This technique could be used on other installations where HIBP systems are installed. The hardware for these measurements is not very complicated whereas the results obtained are precise and reliable.

The correction values found using this technique in WEGA are included in the ray tracing 
code. This allows improving the coordinate mapping precision. However, in the WEGA case the correction of the ionisation point mapping is comparable with the spatial resolution of the HIBP measurements. Therefore, the correction of the calculations has a minor influence on the final measurement results.

Generally, calibration of the HIBP with the 32 plate detector shows a good consistency of the ray tracing calculation model and allows identifying some minor discrepancies in the HIBP assembly. There is no need to adjust deflecting plates and the primary beam line position as far as the corrections are included in the ray tracing calculations. Thus the calculated values reflect the real situation at WEGA.

However, the calibration should be performed each time after a maintenance of the HIBP. For example, each time when the ion source working body is replaced by a new one. The measurements should be repeated because changing the ion source configuration could influence the initial distribution of the ions over the working body surface. This would lead, as a consequence, to a deflection of the primary ion beam from the calculated trajectory in radial or/and toroidal direction, which is not the case for currently installed ion source.

\section{REFERENCES}

${ }^{1}$ T. Ido, A. Shimizu, M. Nishiura, A. Nishizawa, S. Katoh, K. Tsukada, M. Yokota, H. Ogawa, T. Inoue, and Y. Hamada, Rev. Sci. Instrum. 77, 10F523 (2006)

${ }^{2}$ L. I. Krupnik, I.S. Bondarenko, A.A. Chmyga, M.B. Dreval, S.M. Khrebtov, A.D. Komarov , A.S. Kozachok , C. Hidalgo, I. Garcia-Cortes, L. Rodriguez-Rodrigo, A.V. 
Melnikov, P. Coelho, M. Cunha, B. Goncalves, A. Malaquias, I.S. Nedzelskiy, C.A.F. Varandas, Fusion Eng. Des. 56-57, 935 (2001)

${ }^{3}$ F. C. Jobes, J. F. Marshall and R. L. Hickok, Phys. Rev. Lett., vol. 22, p. 1042, 1969.

${ }^{4}$ L. I. Krupnik, G. N. Deshko, A. I. Zhezhera, A. A. Chmyga, A. D. Komarov, A. S.

Kozachek, A. V. Melnikov, S. V. Perfilov, M. Otte, M. Shubert, Fusion Science and Technology 50, (2006) 276-280

${ }^{5}$ Y. Podoba, M. Otte, F. Wagner, I. Bondarenko, A. Chmyga, G. Deshko, A. Komarov, A. Kozachek, L. Krupnik, S. Khrebtov, A. Zhezhera, A. Melnikov, S. Perfilov, and M. Schubert, AIP Conf. Proc. March 19, 2008 V. 993, pp. 235-238

${ }^{6}$ M. Otte, D. Andruczyk, E. Holzhauer, J. Howard, R. König, L. Krupnik, H. P. Laqua, O. Lischtschenko, S. Marsen, J. Schacht, J. Urban, Y. Y. Podoba, J. Preinhalter, F. Wagner, G. B. Warr, and A. Zhezhera, PLASMA 2007 October 16-19, AIP Conf. Proc. March 19, 2008 V. 993, pp. 3-10

${ }^{7}$ G. B. Warr, H. P. Laqua, D. Assmus, W. Kasparek, D. Keil, O. Lischtschenko, S. Marsen, M. Otte, M. Schubert, F. Wagner, 33rd EPS Conference on Plasma Phys. Rome, 19 - 23 June 2006 ECA Vol.30I, P-2.122 (2006)

${ }^{8}$ H.P. Laqua, D. Andruczyk, S. Marsen, M. Otte, J. Preinhealter, J. Urban, G.B. Warr, D. Zhang, Proc. of the 22nd IAEA Fusion Energy Conference Geneva, 13-18 October 2008, $\mathrm{PD} / \mathrm{P} 1-7$

${ }^{9}$ T.S.Green and G.A.Proca, Rev.Sci. Instr. 41, 1409 (1970)

10 J. J. Zielinski, S. C. Aceto, J. G. Schwelberger, K. A. Connor, J. F. Lewis, J. C. Glowienka, A. Carnevali, Rev. Sci. Instrum. 63, 4574 (1992) 
11 K. Kamiya, Y. Miura, K. Tsuzuki, T. Ido, Y. Hamada, T. Nakayama, Rev. Sci. Instrum. 72, 579 (2001)

${ }^{12}$ S. Lee, A. Fujisawa, T. P. Crowley, H. Iguchi, M. Kojima, Y. Hamada, K. Nishimura, S. Okamura and K. Matsuoka, Fusion Engineering and Design 34-35 (1997)645-648

${ }^{13}$ J. Lei, U. Shah, D. R. Demers, K. A. Connor, P. M. Schoch, Rev. Sci. Instrum. 72, 564 (2001)

${ }^{14}$ F. M. Bieniosek, P. L. Colestock, K. A. Connor, R. L. Hickok, S. P. Kuo, R. A. Dandl, Rev. Sci. Instrum. 51, 206 (1980)

${ }^{15}$ K. Takasugi, H. Iguchi, M. Fujiwara, H. Ikegami, Jpn. J. Appl. Phys. 23 (1984) pp. 364-372 
WEGA

vacuum vessel

magnetic flux surfaces

magnetic coils

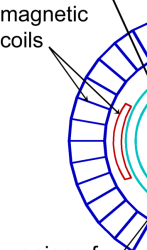

region of detector measurements electrostatic deflecting plates

Faraday ion source

Primary beam line Colp wire grid
detector 

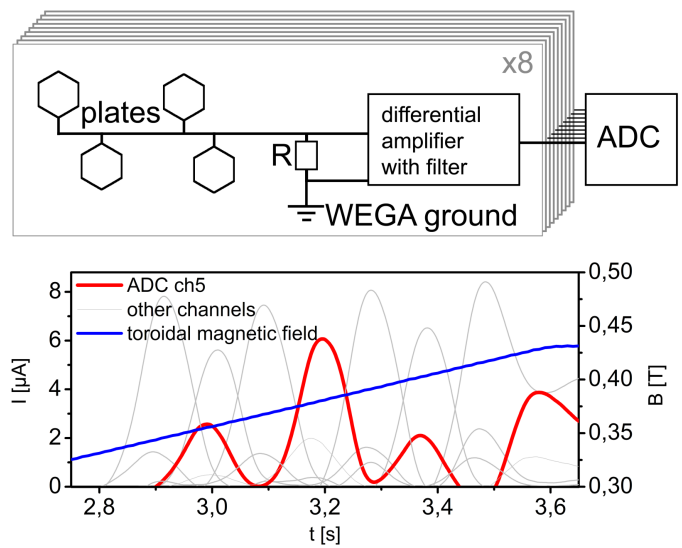


\section{$X[\mathrm{~mm}]$}

\section{$16.5 \quad 22.5 \quad 28.6 \quad 34.6 \quad 40.5 \quad 46.5 \quad 52.3 \quad 58.6 \quad 64.4$}

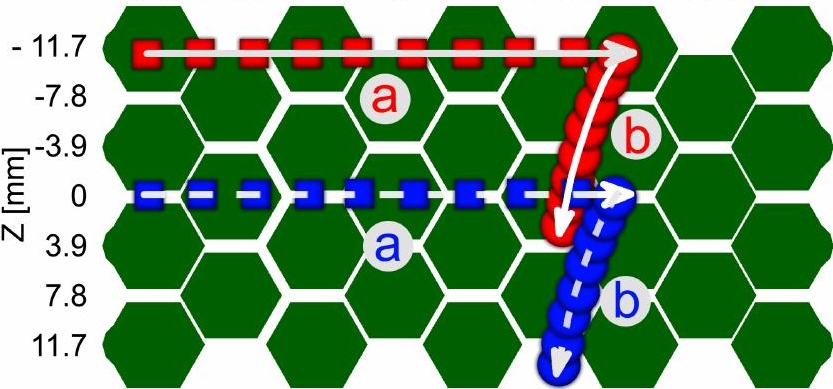

-168.2 -167.6 -166.5 -165.5 -164.0 -162.5 -160.6 -158.2 -156.0

$\beta 1=0 \mathrm{~V}$

$\mathrm{Y}[\mathrm{mm}]$

a: $I_{t}=0 . .3400 \mathrm{~A}, \mathrm{I}_{\mathrm{h}}=0 \mathrm{~A}$

b: $I_{t}=3400 A, I_{h}=0 . .4600 A$

\section{$X[\mathrm{~mm}]$}

$\begin{array}{lllllllll}16.5 & 22.5 & 28.6 & 34.6 & 40.5 & 46.5 & 52.3 & 58.6 & 64.4\end{array}$

-
$\frac{E}{E}$
$N$

$-7.8$

$-3.9$

0

3.9

7.8

11.7
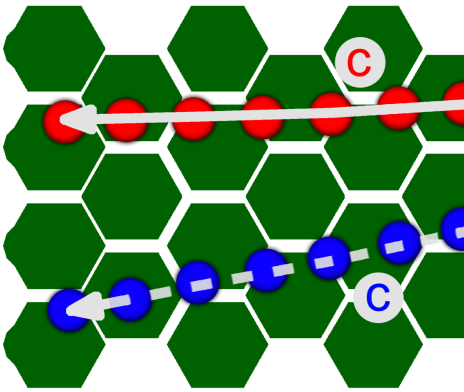

-168.2 -167.6 -166.5 -165.5 -164.0 -162.5 -160.6 -158.2 -156.0

$\beta 1=-130 \mathrm{~V}$

$\mathrm{Y}[\mathrm{mm}]$

c: $I_{t}=3000 \mathrm{~A}, I_{h}=4200 \mathrm{~A}, \alpha 2=0 . .4 \mathrm{kV}$ 


\section{(b)}

deflecting plates

horizontal

misalignment

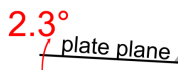

\section{horizontal plane}

beam

(a) $0.4^{\circ}$

- $77 / 2 \mathrm{~m} / \mathrm{m}$

- primary beam-line

toroidal insertion angle misalignment

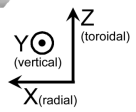

